\title{
Anti-arrhythmic effects of hypercalcemia in hyperkalemic, Langendorff-perfused mouse hearts
}

\author{
GARY TSE ${ }^{1 *}$, BING SUN $^{2 *}$, SHEUNG TING WONG $^{3}$, VIVIAN TSE $^{4}$ and JIE MING YEO ${ }^{3}$ \\ ${ }^{1}$ School of Biomedical Sciences, Li Ka Shing Faculty of Medicine, University of Hong Kong, Hong Kong, SAR; \\ ${ }^{2}$ Department of Cardiology, Tongji University Affiliated Tongji Hospital, Shanghai 200065, P.R. China; \\ ${ }^{3}$ School of Medicine, Imperial College London, London SW7 2AZ, UK; ${ }^{4}$ Department of \\ Physiology, McGill University, Montreal, Quebec H3G 1Y6, Canada
}

Received March 24, 2016; Accepted May 31, 2016

DOI: $10.3892 /$ br.2016.735

\begin{abstract}
The present study examined the ventricular arrhythmic and electrophysiological properties during hyperkalemia $\left(6.3 \mathrm{mM}\left[\mathrm{K}^{+}\right]\right.$vs. $4 \mathrm{mM}$ in normokalemia) and anti-arrhythmic effects of hypercalcemia $\left(2.2 \mathrm{mM}\left[\mathrm{Ca}^{2+}\right]\right)$ in Langendorff-perfused mouse hearts. Monophasic action potential recordings were obtained from the left ventricle during right ventricular pacing. Hyperkalemia increased the proportion of hearts showing provoked ventricular tachycardia (VT) from 0 to 6 of 7 hearts during programmed electrical stimulation (Fisher's exact test, $\mathrm{P}<0.05$ ). It shortened the epicardial action potential durations $\left(\mathrm{APD}_{\mathrm{x}}\right)$ at $90,70,50$ and $30 \%$ repolarization and ventricular effective refractory periods (VERPs) (analysis of variance, $\mathrm{P}<0.05$ ) without altering activation latencies. Endocardial $\mathrm{APD}_{\mathrm{x}}$ and VERPs were unaltered. Consequently, $\triangle \mathrm{APD}_{\mathrm{x}}$ (endocardial $\mathrm{APD}_{\mathrm{x}}$-epicardial $\mathrm{APD}_{\mathrm{x}}$ ) was increased, VERP/latency ratio was decreased and critical intervals for reexcitation $\left(\mathrm{APD}_{90}-\mathrm{VERP}\right)$ were unchanged. Hypercalcemia treatment exerted anti-arrhythmic effects during hyperkalemia, reducing the proportion of hearts showing VT to 1 of 7 hearts. It increased epicardial VERPs without further altering the remaining parameters, returning VERP/latency ratio to normokalemic values and also decreased the critical intervals. In conclusion, hyperkalemia exerted pro-arrhythmic effects by shortening APDs and VERPs. Hypercalcemia exerted anti-arrhythmic effects by reversing VERP changes, which scaled the VERP/latency ratio and critical intervals.
\end{abstract}

Correspondence to: Dr Gary Tse, School of Biomedical Sciences, Li Ka Shing Faculty of Medicine, University of Hong Kong, 3/F Laboratory Block, 21 Sassoon Road, Hong Kong, SAR, P.R. China

E-mail: gary.tse@doctors.org.uk

${ }^{*}$ Contributed equally

Key words: hyperkalemia, hypercalcemia, ventricular arrhythmia, action potential duration

\section{Introduction}

The extracellular potassium concentration $\left(\left[\mathrm{K}^{+}\right]_{0}\right)$ is normally maintained between 3.5 and $5 \mathrm{mM}$. Hyperkalemia and hypokalemia are defined as a serum potassium concentration above and below this range, respectively (1). The two are important clinical conditions (2), predisposing patients to life-threatening ventricular arrhythmias $(3,4)$. Of these, hyperkalemia exerts a wide range of effects on cardiac conduction and repolarization properties, depending on the degree of high $\left[\mathrm{K}^{+}\right]_{0}$. Its most common electrocardiographic manifestations are flattened or loss of the P-wave (5), prolonged PR and QRS intervals (6), and T-wave abnormalities, particularly peaked T-waves (7). A sine-wave appearance can be observed at the most severely elevated levels of $\left[\mathrm{K}^{+}\right]_{0}(8)$. Calcium gluconate or $10 \%$ calcium chloride are used acutely to suppress ventricular arrhythmias in hyperkalemic patients $(9,10)$, despite the fact that hypercalcemia alone has pro-arrhythmic effects $(11,12)$. There have been certain previous studies on the electrophysiological changes during hyperkalemia $(13,14)$, but not on the mechanism underlying the anti-arrhythmic action of calcium in this situation, apart from its 'membrane-stabilizing effect' (15). This notion has been disputed and the protective action of high $\left[\mathrm{Ca}^{2+}\right]_{0}$ has instead been attributed to restoration of conduction velocities (CVs) back to normal values (16).

Mouse systems have been extensively used for the study of arrhythmogenesis, as they permit the use of genetic and pharmacological manipulation to produce ion channel abnormalities with great translational potential (17-26). This has resulted in demonstrations of the following mechanisms $(27,28)$. Firstly, the early-after depolarization phenomena and triggered activity observed during hypokalemia have been attributed to prolonged action potential durations (APDs) (29). Secondly, several reentrant substrates during hypokalemia have been identified: Prolonged epicardial but unaltered endocardial APDs leading to negative $\triangle \mathrm{APD}_{90}$ given by endocardial $\mathrm{APD}_{90}$-epicardial $\mathrm{APD}_{90}(30)$. Reduced ventricular effective refractory periods (VERPs) leading to increased critical intervals given by $\mathrm{APD}_{90}$-VERP (29). By contrast, reduced CVs were shown to induce ventricular arrhythmias following treatment with the gap junction and sodium channel inhibitor heptanol through 
a reduction in excitation wavelengths despite unaltered APDs and even with increased VERPs $(31,32)$. However, to the best of our knowledge, there have been no investigations of the arrhythmogenic effects of hyperkalemia in the mouse system.

Therefore, in the present study, the ventricular arrhythmogenic properties of hyperkalemia were characterized in Langendorff-perfused mouse hearts for the first time. An increased external calcium concentration is known to reduce membrane excitability at the cellular level (33), but exerts pro-arrhythmic effects in the whole heart level under normokalemic conditions (34). However, as a decrease in membrane excitability would lead to an increase in refractoriness, it was hypothesized that hypercalcemia would abolish arrhythmic properties of hyperkalemia by increasing VERPs.

\section{Materials and methods}

Solutions. Krebs-Henseleit solution $[119 \mathrm{mM} \mathrm{NaCl}, 25 \mathrm{mM}$ $\mathrm{NaHCO}_{3}, 4 \mathrm{mM} \mathrm{KCl}, 1.2 \mathrm{mM} \mathrm{KH_{2 }} \mathrm{PO}_{4}, 1 \mathrm{mM} \mathrm{MgCl}$, $1.8 \mathrm{mM} \mathrm{CaCl}_{2}, 10 \mathrm{mM}$ glucose and $2 \mathrm{mM}$ sodium pyruvate (pH 7.4)] that had been bicarbonate-buffered and bubbled with $95 \% \mathrm{O}_{2}-5 \% \mathrm{CO}_{2}$ (35) was used in the experiments. Hyperkalemic solution was prepared by increasing the amount of $\mathrm{KCl}$ added to produce a $\left[\mathrm{K}^{+}\right]$of $6.3 \mathrm{mM}$, whereas hypercalcemic solution was prepared by increasing the amount of $\mathrm{CaCl}_{2}$ added to produce a $\left[\mathrm{Ca}^{2+}\right]$ of $2.2 \mathrm{mM}$.

Preparation of Langendorff-perfused mouse hearts. Wild-type mice of the 129 genetic background between 5 and 7 months of age were used in the study. These mice were housed in an animal facility at room temperature $\left(21 \pm 1^{\circ} \mathrm{C}\right)$, subject to a $12: 12 \mathrm{~h}$ light:dark cycle and had free access to sterile rodent chow and water. All the experiments described complied with the UK Animals (Scientific Procedures) Act 1986. The procedures for the preparation of Langendorff-perfused mouse hearts have been described previously (36). Mice were sacrificed by cervical dislocation in accordance with Sections 1(c) and 2 of Schedule 1 of the UK Animals (Scientific Procedures) Act 1986. The hearts were quickly excised and immediately submerged in ice-cold Krebs-Henseleit solution. The aorta was cannulated using a tailor-made 21-gauge cannula that had been prefilled with ice-cold buffer, secured using a micro-aneurysm clip (Harvard Apparatus, Cambridge, UK) and attached to the perfusion system. Retrograde perfusion was started at a rate of 2-2.5 ml min ${ }^{-1}$ using a peristaltic pump (Watson-Marlow Bredel pumps model 505S; Falmouth, Cornwall, UK) with the perfusate passing through 200 - and $5-\mu \mathrm{m}$ filters successively and heated to $37^{\circ} \mathrm{C}$ using a water jacket and circulator prior to reaching the aorta. Approximately $90 \%$ of the hearts that regained their pink colour and spontaneous rhythmic activity were studied further. The remaining $10 \%$ did not and were therefore discarded. Perfusion continued for a further $20 \mathrm{~min}$ to minimise any residual effects of endogenous catecholamine release prior to examination of the electrophysiology of the perfused hearts.

Stimulation protocols. Electrical stimulation was achieved using paired platinum electrodes ( $1 \mathrm{~mm}$ interpole distance) placed at the basal right ventricular epicardium. Pacing
A

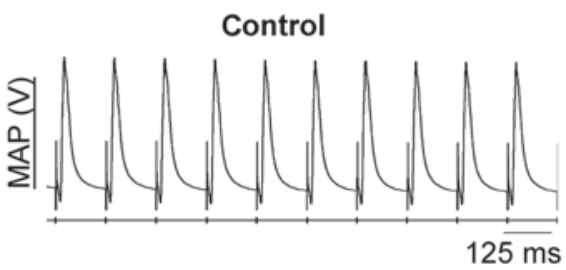

B hyper $\mathrm{K}^{+}$

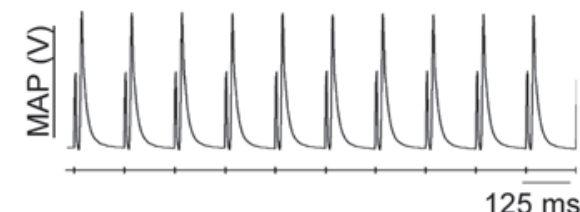

C hyper $\mathrm{K}^{+}+\mathrm{Ca}^{2+}$

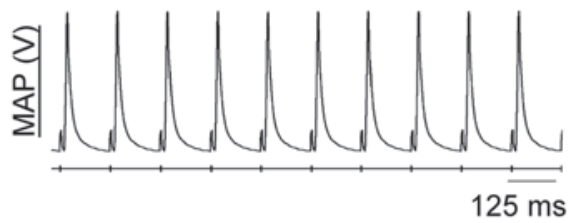

Figure 1. Representative epicardial MAP recordings obtained under (A) control conditions, (B) hyperkalemia alone or (C) following hypercalcemia treatment during regular $8 \mathrm{~Hz}$ pacing. Typical regular rhythms can be observed, with each MAP occurring directly following its preceding stimulus. MAP, monophasic action potential.

occurred at $8 \mathrm{~Hz}$, using square wave pulses $2 \mathrm{msec}$ in duration, with a stimulation voltage set to three times the diastolic threshold (Grass S48 Stimulator; Grass-Telefactor, Slough, UK) immediately after the start of perfusion. This allowed direct comparisons with previous mouse studies of arrhythmogenesis (29-32). Programmed electrical stimulation (PES) was used to assess for arrhythmogenicity and thereby for reentrant substrates. This procedure consisted of a drive train of eight regularly paced S1 stimuli at a $125 \mathrm{msec}$ baseline cycle length (BCL), followed by premature $\mathrm{S} 2$ extra-stimuli every ninth stimulus. S1S2 intervals first equalled the pacing interval and were successively reduced by $1 \mathrm{msec}$ with each nine stimulus cycle until arrhythmic activity was initiated or refractoriness was reached, whereupon the $\mathrm{S} 2$ stimulus elicited no response.

Recording procedures. Monophasic action potentials (MAPs) recordings were obtained from the left ventricular epicardium using an MAP electrode (Linton Instruments, Harvard Apparatus). They were also obtained from the left endocardium using a custom-made MAP electrode that was made from two strands of $0.25-\mathrm{mm}$ Teflon-coated silver wire (99.99\% purity; Advent Research Materials, Witney, UK). The tips of the electrode had previously undergone galvanic treatment with chloride to eliminate DC offset. The endocardial electrode was introduced through a small access window made in the inter-ventricular septum and subsequently positioned on the lateral aspect of the left ventricular cavity. All the recordings were performed using a $\mathrm{BCL}$ of $125 \mathrm{msec}(8 \mathrm{~Hz})$ to exclude rate-dependent differences in APDs. MAPs were pre-amplified using an NL100AK head stage, amplified with an NL104A amplifier and band-pass filtered between $0.5 \mathrm{~Hz}$ and $1 \mathrm{kHz}$ using an NL125/6 filter (Neurolog, Hertfordshire, UK) and subsequently digitized (1401plus MKII; Cambridge 
A

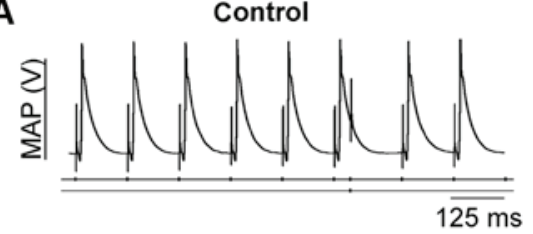

B hyper $\mathrm{K}^{+}$
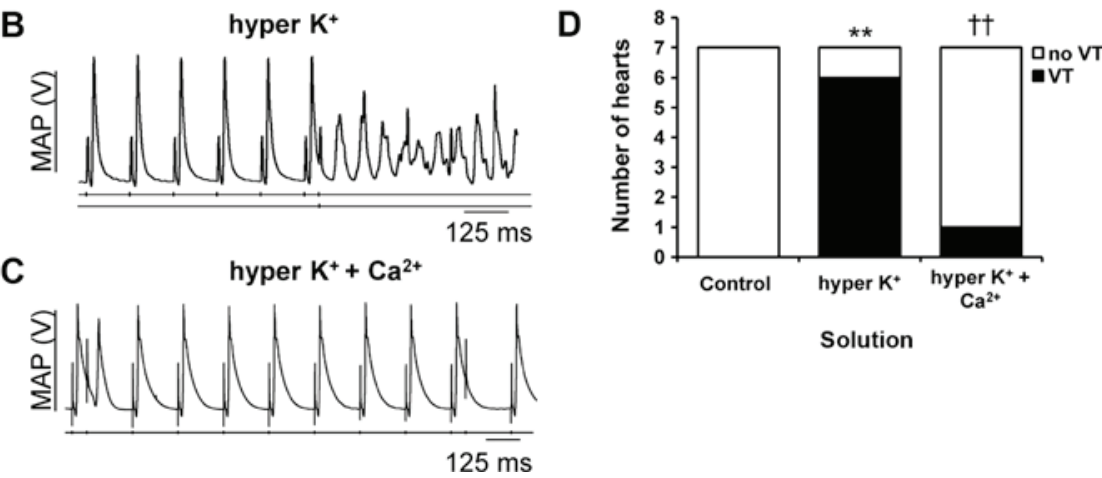

Figure 2. Representative epicardial MAP recordings from programmed electrical stimulation (PES) under (A) control conditions, and (B) hyperkalemia alone and (C) following hypercalcemia treatment. (D) The incidences of provoked ventricular tachycardia (VT) demonstrate the pro-arrhythmic effects of hyperkalemia (Fisher's exact test, $\left.{ }^{* *} \mathrm{P}<0.01\right)$ and the subsequent anti-arrhythmic effects of hypercalcemia treatment (Fisher's exact test, $\left.{ }^{\circ} \mathrm{P}<0.01\right)$. MAP, monophasic action potential.

Electronic Design, Cambridge, UK) at $5 \mathrm{kHz}$. Following this, they were analyzed using Spike2 software (Cambridge Electronic Design). MAP waveforms that did not match the previously established stringent criteria for MAP signals (37) were rejected. The MAPs must have stable baselines, fast upstrokes, with no inflections or negative spikes, and a rapid first phase of repolarization. The peak of the MAP was used to measure $0 \%$ repolarization and $100 \%$ repolarization was measured at the point of return of the potential to baseline (37-39). The following parameters were measured: Activation latency, defined as the time difference between the stimulus and the peak of the MAP; $\mathrm{APD}_{\mathrm{x}}$, defined as the time difference between the peak of the MAP and $\mathrm{x} \%$ repolarization; and VERP.

Statistical analysis. All the values are expressed as mean \pm standard error of the mean. Different experimental groups were compared by one-way analysis of variance (ANOVA) and Student's t-test was used as appropriate. $\mathrm{P}<0.05$ was considered to indicate a statistically significant difference. Categorical data were compared with Fisher's exact test (one-tailed).

\section{Results}

Ventricular arrhythmogenicity and action potential characteristics. Ventricular arrhythmogenicity and its associations to action potential characteristics were examined under normokalemia $\left(5.2 \mathrm{mM}\left[\mathrm{K}^{+}\right]\right)$, normocalcemia $\left(1.8 \mathrm{mM}\left[\mathrm{Ca}^{2+}\right]\right)$, hyperkalemia alone $\left(6.3 \mathrm{mM}\left[\mathrm{K}^{+}\right]\right)$and hyperkalemia with hypercalcemia treatment $\left(2.2 \mathrm{mM}\left[\mathrm{Ca}^{2+}\right]\right)$.

Hyperkalemia exerts pro-arrhythmic effects that are abolished by hypercalcemia. The initial experiments were performed on hearts extrinsically paced at $8 \mathrm{~Hz}$, which is close to the heart rate observed in vivo under normokalemic, hyperkalemic and combined hyperkalemic and hypercalcemic conditions. Fig. 1 shows representative traces of epicardial MAP recordings under these pharmacological conditions, in which stable MAPs occurring directly following its preceding stimulus, with consistent waveforms, can be observed.

PES delivering progressively premature stimuli was used to examine the arrhythmic tendency. It consistently failed to provoke any arrhythmia under the control conditions (Fig. 2A; S2 extrastimulus indicated by an arrow). By contrast, provoked ventricular tachycardia (VT) was observed under hyperkalemic conditions alone (Fig. 2B). This was prevented by further hypercalcemia treatment (Fig. 2C). The incidences of provoked VT observed are summarized in Fig. 2D, demonstrating that hyperkalemia was significantly arrhythmogenic (Fisher's exact test, $\mathrm{P}<0.01$ ), whereas hypercalcemia treatment was anti-arrhythmic under hyperkalemic conditions (Fisher's exact test, $\mathrm{P}<0.01$ ).

Shortenings in the QT interval were observed in electrocardiograms (ECGs) obtained from patients suffering from hyperkalemia (6). This may reflect alterations in APD either locally or transmurally across the myocardial wall. APDs at $\mathrm{x}=30,50,70$ and $90 \%$ repolarization $\left(\mathrm{APD}_{\mathrm{x}}\right)$ were therefore assessed in the epicardium and endocardium, allowing calculation of $\triangle \mathrm{APD}_{90}$ given by endocardial $\mathrm{APD}_{90}$-epicardial $\mathrm{APD}_{90}$, thereby providing an indication of the transmural repolarization gradient. Epicardial $\mathrm{APD}_{90}$ was decreased from 42.2 \pm 2.6 to $24.5 \pm 1.6 \mathrm{msec}$ by hyperkalemia $(\mathrm{P}<0.001$; Fig. $3 \mathrm{~A})$, as were $\mathrm{APD}_{70}\left(\mathrm{P}<0.001\right.$; Fig. 3B), $\mathrm{APD}_{50}\left(\mathrm{P}<0.01\right.$; Fig. 3C) and $\mathrm{APD}_{30}$ $(\mathrm{P}<0.05$; Fig. 3D). However, the corresponding endocardial $\mathrm{APD}_{\mathrm{x}}$ values were not altered $(\mathrm{P}>0.05$; Fig. $3 \mathrm{E}-\mathrm{H})$. These changes corresponded to increases in $\triangle \mathrm{APD}_{90}$ (Student's t-test, $\mathrm{P}<0.05$; Fig. 4A), $\triangle \mathrm{APD}_{70}\left(\mathrm{P}<0.01\right.$; Fig. 4B), $\Delta \mathrm{APD}_{50}(\mathrm{P}<0.01$; Fig. $4 \mathrm{C})$ and $\triangle \mathrm{APD}_{30}(\mathrm{P}<0.05 ;$ Fig. $4 \mathrm{D})$. None of the epicardial or endocardial $\mathrm{APD}_{\mathrm{x}}$ and $\triangle \mathrm{APD}_{\mathrm{x}}$ values were further altered upon hypercalcemia treatment ( $\mathrm{P}>0.05$ in all cases).

Epicardial VERPs were decreased from $45.9 \pm 1.7$ to $33.7 \pm 2.6$ msec during hyperkalemia (ANOVA, $\mathrm{P}<0.001$; Fig. $5 \mathrm{~A}$ ) 


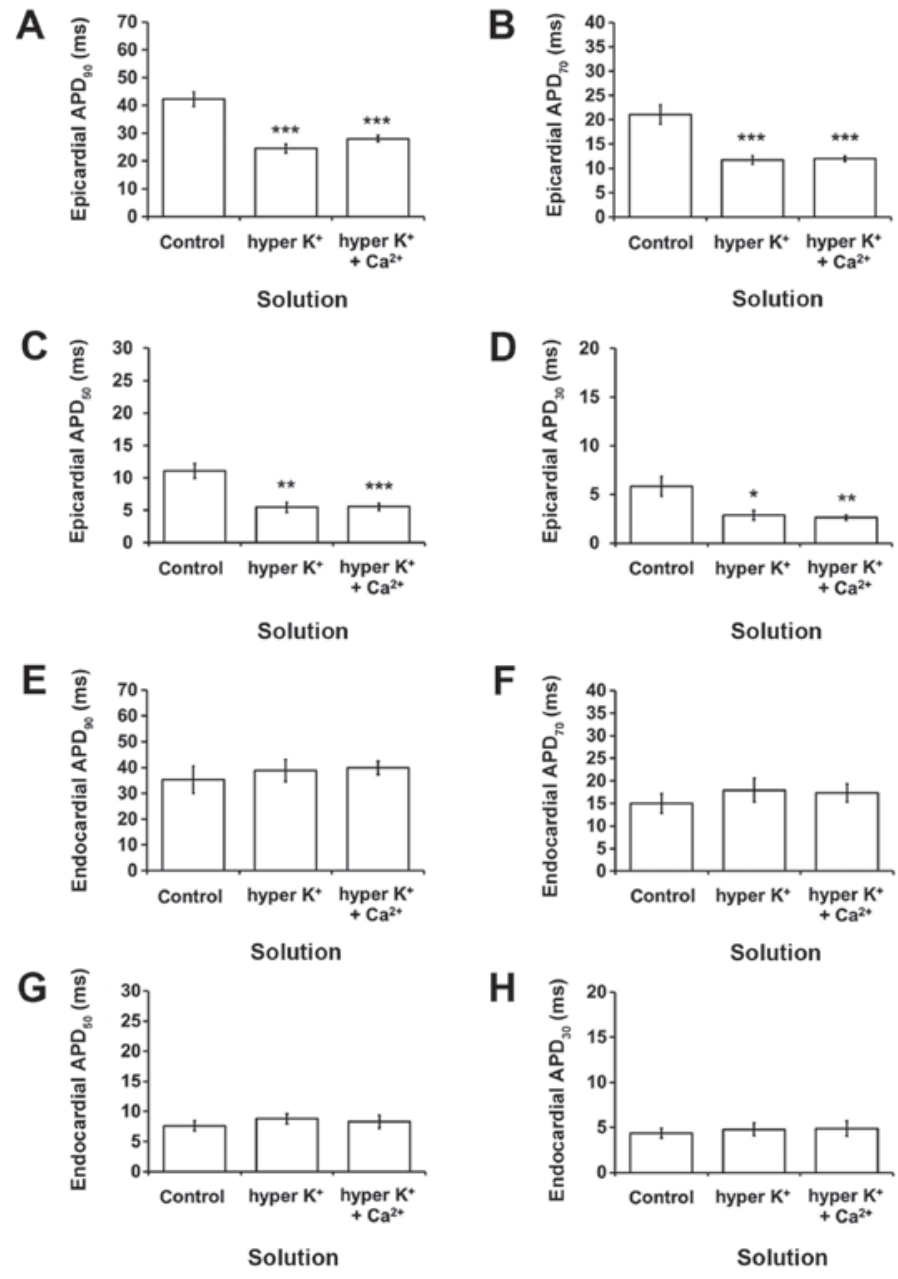

Figure 3. Epicardial action potential durations $\left(\mathrm{APD}_{\mathrm{x}}\right)$ at $\mathrm{x}=(\mathrm{A})$ 90, (B) 70, (C) 50 and (D) 30\% repolarization (msec) (mean $\pm \mathrm{SEM}$ ) (C) under control conditions, hyperkalemia alone or following hypercalcemia treatment during $8 \mathrm{~Hz}$ pacing $(\mathrm{n}=7)$. All $\mathrm{APD}_{\mathrm{x}}$ values were shortened by hyperkalemia $(\mathrm{ANOVA}$, ${ }^{* * * *} \mathrm{P}<0.001,{ }^{* * * *} \mathrm{P}<0.001,{ }^{* * *} \mathrm{P}<0.01,{ }^{*} \mathrm{P}<0.05$, respectively), which were not further altered by hypercalcemia treatment $(\mathrm{ANOVA} \mathrm{P}>0.05)$. Endocardial $A P D_{\mathrm{x}}$ at $\mathrm{x}=(\mathrm{E})$ 90, (F) 70, (G) 50 and (H) 30\% repolarization (msec) (mean \pm SEM) obtained under the same experimental conditions. None of these values was altered by hyperkalemia alone or following hypercalcemia treatment (ANOVA, P>0.05). APD, action potential duration; SEM, standard error of the mean; ANOVA, analysis of variance.

A

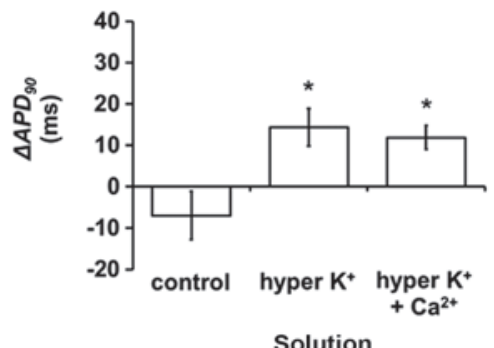

C

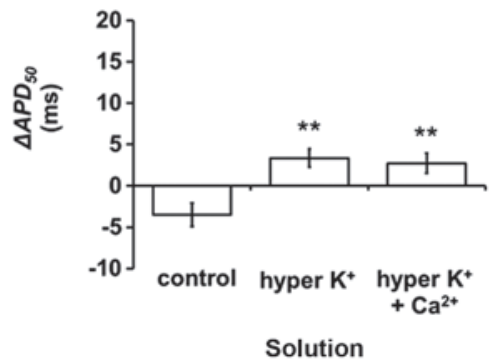

B

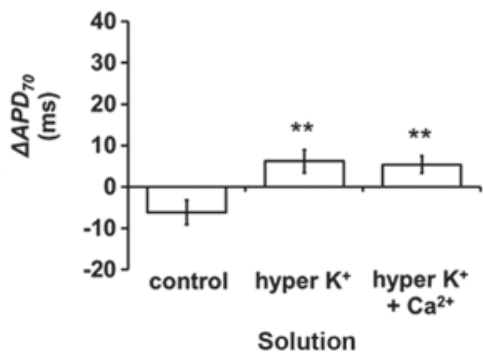

D

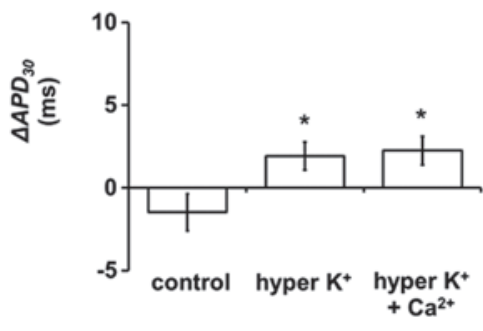

Solution

Figure 4. $\triangle \mathrm{APD}_{\mathrm{x}}$ (endocardial $\mathrm{APD}_{\mathrm{x}}$-epicardial $\mathrm{APD}_{\mathrm{x}}$ ) at $\mathrm{x}=(\mathrm{A}) 90$, (B) 70 , (C) 50 and (D) $30 \%$ repolarization (msec) (mean \pm standard error of the mean) under control conditions, hyperkalemia alone or after hypercalcemia treatment during $8 \mathrm{~Hz}$ pacing $(\mathrm{n}=7)$. $\triangle \mathrm{APD}_{90}, \triangle \mathrm{APD}_{70}, \Delta \mathrm{APD}_{50}$ and $\triangle \mathrm{APD}_{30} \mathrm{were}$ increased by hyperkalemia (Student's t-test, ${ }^{*} \mathrm{P}<0.05,{ }^{* *} \mathrm{P}<0.01,{ }^{* *} \mathrm{P}<0.01$ and ${ }^{*} \mathrm{P}<0.05$, respectively) and were not further altered by hypercalcemia treatment (P $\left.>0.05\right)$. APD, action potential duration. 
and reversed by hypercalcemia treatment $(\mathrm{P}>0.05)$. By contrast, endocardial VERPs had a mean value of $36.7 \pm 2.1 \mathrm{msec}$ under control conditions and this was not altered by hyperkalemia alone or following hypercalcemia treatment ( $\mathrm{P}>0.05$; Fig. 5B). Epicardial VERPs were significantly shorter compared to the corresponding endocardial VERPs under hyperkalemic conditions alone $(\mathrm{P}<0.05)$ but not under control conditions or hyperkalemic conditions following hypercalcemia treatment ( $\mathrm{P}>0.05)$.

Hyperkalemia is known to cause prolongations in QRS durations, reflecting slowed ventricular conduction in humans (6). Reduced CVs have been shown to be an important factor in producing ventricular arrhythmogenesis following heptanol treatment (31). Therefore, in the studythe activation latencies, which provide an indication of the CVs, were quantified to determine whether changes in these values contribute to the arrhythmogenic substrate. Epicardial and endocardial activation latencies had values of $16.7 \pm 0.8$ (Fig. 6A) and $17.0 \pm 1.1 \mathrm{msec}$ (Fig. 6B), respectively, under normokalemic conditions. These values were not altered by hyperkalemia alone or following hypercalcemia treatment (ANOVA, P $>0.05$ ). Epicardial activation latencies were not significantly different from their corresponding endocardial activation latencies under any of the aforementioned pharmacological conditions studied $(\mathrm{P}>0.05)$.

Increased critical intervals for reexcitation have previously been associated with increased arrhythmogenicity in hypokalemic mouse hearts (40). To determine their possible roles in hyperkalemia-induced arrhythmogenesis, these values were accordingly calculated for all the pharmacological conditions studied. The local critical interval for the epicardium was $-7.0 \pm 4.1 \mathrm{msec}$ under control conditions ( $\mathrm{n}=7$; Fig. $7 \mathrm{~A})$. The interval was not altered by hyperkalemia alone but was reduced by hypercalcemia treatment to $-23.1 \pm 4.5 \mathrm{msec}$ (ANOVA, $\mathrm{P}<0.05)$. By contrast, the local critical interval for the endocardium had a value of $-1.4 \pm 3.5 \mathrm{msec}(\mathrm{n}=7$; Fig. 7B) but this was not altered by either hyperkalemia alone or following further hypercalcemia treatment $(\mathrm{P}>0.05)$. The transmural critical interval for reexcitation of the endocardium by the epicardium had a value of $5.3 \pm 3.5 \mathrm{msec}(\mathrm{n}=7$; Fig. 7C). This was reduced by hyperkalemia to $-16.2 \pm 4.2 \mathrm{msec}$ (Student's t-test, $\mathrm{P}<0.01$ ) and not further altered by hypercalcemia treatment $(\mathrm{P}>0.05)$. By contrast, the critical interval for reexcitation of the epicardium by the endocardium had a value of $-10.3 \pm 5.7 \mathrm{msec}$, and was not altered by either hyperkalemia alone or following hypercalcemia treatment (Fig. 7D; P>0.05).

Reductions in the wavelength of excitation, defined as the product of VERP and CV, increase the likelihood of arrhythmogenesis (41). The VERP/latency can be used as a surrogate marker of wavelength (42). VERP/latency was decreased by hyperkalemia from $2.8 \pm 0.2$ to $1.9 \pm 0.2 \mathrm{~mm}(\mathrm{n}=8$; ANOVA, $\mathrm{P}<0.01$; Fig. 7E) and subsequently restored to $2.8 \pm 0.2 \mathrm{~mm}$ by hypercalcemia treatment, a value that was not statistically different from the control value (ANOVA, P $>0.05$ ).

Such reexcitation criteria employing the concept of the critical interval therefore correlated poorly with arrhythmogenicity in this hyperkalemia model, unlike the case of hypokalemia described previously. This would suggest arrhythmogenesis may not be due to APD exceeding VERP, but may arise from reductions in VERP/latency ratios.
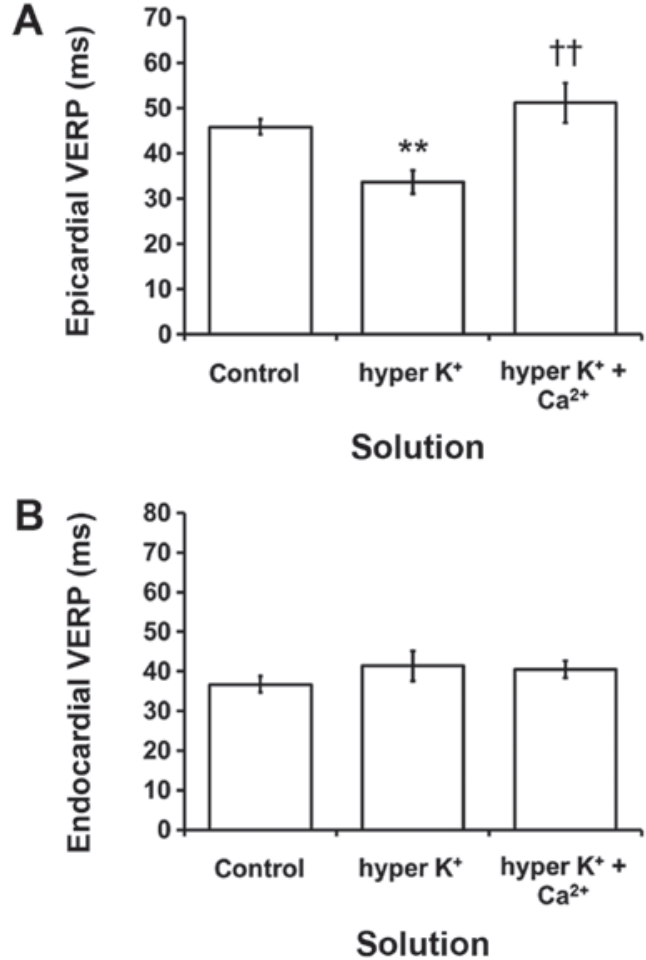

Figure 5. (A) Epicardial and (B) endocardial VERPs obtained under control conditions, hyperkalemia alone or after hypercalcemia treatment $(n=7)$. Hyperkalemia alone reduced epicardial VERP $\left({ }^{* *} \mathrm{P}<0.01\right)$, which was reversed following hypercalcemia treatment $\left({ }^{\dagger} \mathrm{P}<0.01\right)$. Endocardial VERP was not affected by hyperkalemia alone or following hypercalcemia treatment (analysis of variance, $\mathrm{P}>0.05$ ). Epicardial VERPs were significantly shorter compared to the corresponding endocardial VERPs during hyperkalemia alone $(\mathrm{P}<0.05)$ but not under control conditions or hyperkalemic conditions following hypercalcemia treatment $(\mathrm{P}>0.05)$. VERP, ventricular effective refractory periods.
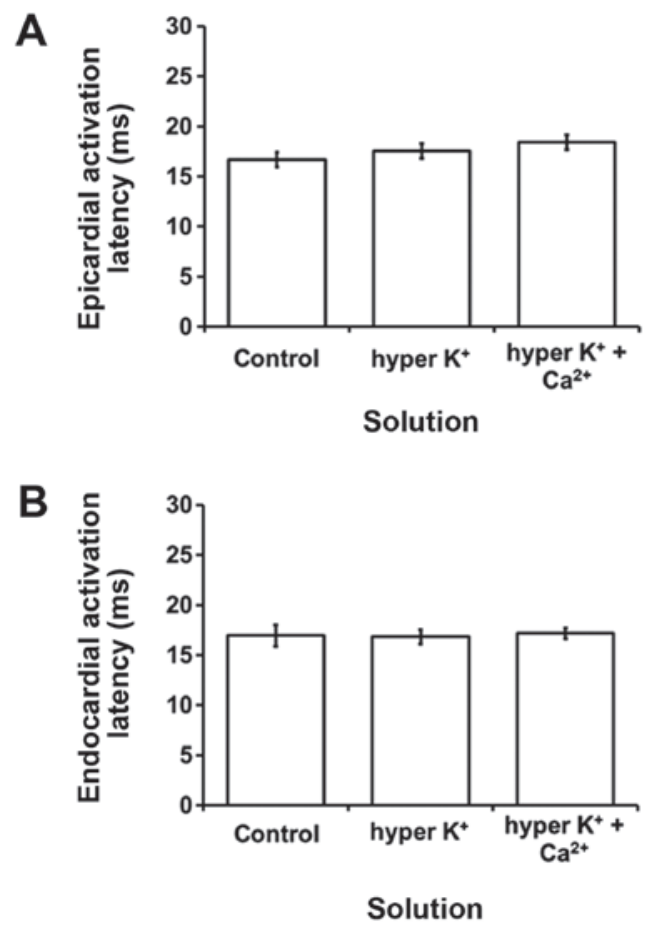

Figure 6. (A) Epicardial and (B) endocardial activation latency obtained under control conditions, hyperkalemia alone or following hypercalcemia treatment $(n=7)$. These values were not altered by hyperkalemia alone or following hypercalcemia treatment (analysis of variance, $\mathrm{P}>0.05$ ). 

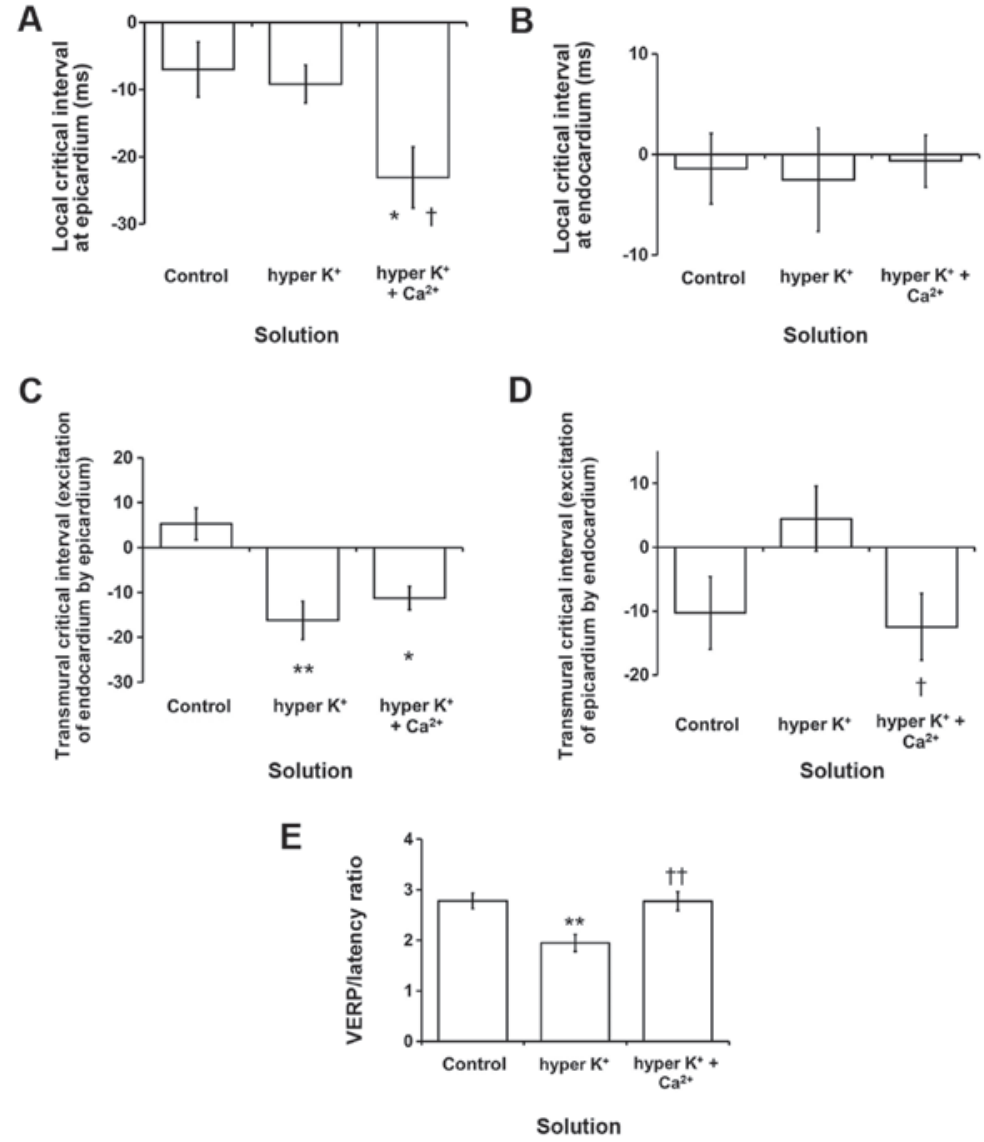

Figure 7. (A-D) Critical intervals $\left(\mathrm{APD}_{90}\right.$-VERP) and (E) VERP/latency ratio. ${ }^{*}$ and ${ }^{* *}$ indicate significant differences from control values, and $\dagger$ indicates significant differences from values obtained during hyperkalemia alone. Local critical intervals obtained from the (A) epicardium were not significantly affected by hyperkalemia alone but were reduced by hypercalcemia treatment (ANOVA, ${ }^{*}, \mathrm{P}<0.05$ ). The local critical interval obtained from the (B) endocardium was not altered by either hyperkalemia alone or following hypercalcemia treatment $(\mathrm{P}>0.05)$. The transmural critical interval for reexcitation of the endocardium by the $(C)$ epicardium was reduced (Student's t-test, ${ }^{*} \mathrm{P}<0.05$ and $\left.{ }^{* *} \mathrm{P}<0.01\right)$ and not further altered by hypercalcemia treatment $(\mathrm{P}>0.05)$. (D) The transmural critical interval for reexcitation of the epicardium by the endocardium was not altered by either hyperkalemia alone or following hypercalcemia treatment ( $\mathrm{P}>0.05$; but there was a difference between $\mathrm{K}^{+}$and $\left.\mathrm{K}^{+}+\mathrm{Ca}^{2+},{ }^{t} \mathrm{P}<0.05\right)$. (E) VERP/latency was decreased by hyperkalemia from $2.8 \pm 0.2$ to $1.9 \pm 0.2 \mathrm{~mm}(\mathrm{n}=8$; ANOVA, ${ }^{* *} \mathrm{P}<0.01 ; \mathrm{K}^{+}$vs. $\left.\mathrm{K}^{+}+\mathrm{Ca}^{2+},{ }^{\dagger} \mathrm{P}<0.01\right)$ and subsequently restored to $2.8 \pm 0.2 \mathrm{~mm}$ by hypercalcemia treatment, a value that was not statistically different when compared to the control value (ANOVA, P $>0.05$ ). APD, action potential duration; VERP, ventricular effective refractory periods; ANOVA, analysis of variance.

\section{Discussion}

Hyperkalemia is one of the most common electrolyte abnormalities observed in hospitalized patients, predisposing them to life-threatening ventricular arrhythmias (43). The mechanisms of arrhythmogenesis have been studied using animal models as they permit the use of genetic or pharmacological manipulation to study the consequences of ion channel abnormalities $(19,20,22,23,44-46)$. In the present study, arrhythmogenic effects of hyperkalemia were examined in Langendorff-perfused mouse hearts. The potential anti-arrhythmic effects of hypercalcemia were also examined under this condition, mimicking $10 \%$ calcium chloride administration used clinically to suppress ventricular arrhythmias in patients suffering from hyperkalemia (10). In the present experiments, epicardial and endocardial MAPs were recorded from the left ventricle during electrical stimulation at the right ventricular epicardium. This led to several new conclusions.

Stable epicardial and endocardial MAP recordings were demonstrated under control conditions, hyperkalemia alone and following hypercalcemia treatment during regular pacing. There was no evidence of spontaneous arrhythmias under these conditions. This subsequently permitted the use of PES to assess arrhythmogenicity and detect the presence of reentrant substrates. No inducible arrhythmias were observed under the control conditions. By contrast, episodes of provoked VT was observed during hyperkalemia, recapitulating clinical findings of increased arrhythmogenicity in humans (9). These arrhythmogenic effects were associated with reductions in the epicardial $\mathrm{APD}_{90}$ and VERP in an absence of alterations in activation latencies, which is inversely proportional to $\mathrm{CV}$. Endocardial $\mathrm{APD}_{90}$ and VERP were not altered. These findings are consistent with the shortened QT intervals observed in ECGs of patients suffering from hyperkalemia (6). The QT interval is a reflection of ventricular repolarization time that is determined by the balance between influx and efflux of ions across the cell membrane (47). Initially, increased $\left[\mathrm{K}^{+}\right]_{0}$ would produce hyperpolarization of the myocardial membrane, but upon reaching a steady state, there is a depolarizing shift in the resting membrane potential (RMP), as described by the Goldman field relationship (48). This has been shown to increase the conductance of the $I_{\mathrm{Kr}}$ channel $(49,50)$, which would accelerate repolarization durations. As well as affecting action potential repolarization, it also influences its initiation 
and subsequent propagation through the myocardium. Thus, hyperkalemia produces a positive shift in the threshold potential (TP) to a smaller extent than the depolarizing shift in RMP, thereby increasing myocardial excitability given by $1 /$ (TP-RMP) $(48,51)$. This could explain why mild hyperkalemia increases CV. However, hyperkalemia also increases the proportion of inactivated sodium channels, reducing $\mathrm{dV} / \mathrm{dt}_{\max }$, and therefore the $\mathrm{CV}$, of the propagating cardiac excitation $(51,52)$. Activation latency was not altered by the hyperkalemia in the present study $\left(\left[\mathrm{K}^{+}\right]_{0}\right.$ of $\left.6.3 \mathrm{mM}\right)$, suggestive of little conduction abnormalities. This may be due to a balance between increased myocardial excitability and reduced proportion of sodium channels available for activation. This is consistent with previous findings that QRS duration was increased or $\mathrm{CV}$ was reduced when $\left[\mathrm{K}^{+}\right]_{\mathrm{o}}$ was $>6.5-7.5 \mathrm{mM}$ (53).

The differing effects of hyperkalemia upon endocardial and epicardial $\mathrm{APD}_{90}$ led to increased $\triangle \mathrm{APD}_{90}$ given by their difference, which is a measure of the transmural repolarization gradient (54). Under normokalemic conditions, the time courses of repolarization are longer in the endocardium compared to the epicardium, giving rise to a positive $\triangle \mathrm{APD}_{90}$. This ensures a normal unidirectional spread of the excitation through the heart $(28,55)$, preventing the epicardium from reexciting the endocardium by phase 2 reentry. It is also responsible for the upright electrocardiographic T-waves in the right precordial leads $(56,57) . \triangle \mathrm{APD}_{90}$ remained positive during exposure to hyperkalemia, suggesting the increased arrhythmogenicity here was not due to reversal of such gradients, as was the case in hypokalemia (29). Instead, the arrhythmogenesis observed can be explained by decreases in the VERP through shortening in the action potential, which would reduce VERP/latency ratios and therefore predispose to reentry (58). However, the critical intervals for reexcitation given by the difference between $\mathrm{APD}_{90}$ and VERP (40) were either unchanged or decreased, which would be expected to have no effect on or decrease, rather than increase, arrhythmogenicity.

Hypercalcemia treatment exerted anti-arrhythmic effects during experimental hyperkalemia, complementing clinical findings that calcium chloride administration is effective in suppressing arrhythmia episodes in hyperkalemic patients (10). It reversed VERP changes without correcting for the shortenings in APDs, and left activation latencies unaltered. Consequently, the VERP/latency ratio returned to control values and the critical intervals were either unchanged or decreased. High $\left[\mathrm{Ca}^{2+}\right]_{0}$ causes a positive shift in the TP without significant effects on the RMP (59). This effect can be explained by adsorption of calcium ions to the outer surface of the cell membrane, generating an electric field that shifts the threshold of $I_{\mathrm{Na}}$ activation to more depolarized potentials (60). It also has a positively inotropic effect in the context of hyperkalemia in a rabbit model (15). Although ventricular tachyarrhythmias attributable to hypercalcemia has been reported in humans (61) and mouse studies (34) under normokalemic conditions, they are nevertheless rare occurrences (62). Due to these protective effects of calcium on the heart, it has been used clinically to treat patients with hyperkalemia acutely prior to correcting for the plasma levels of potassium through the use of insulin and glucose infusion with nebulized salbutamol (63). Notably, no further APD shortening was found in the presence of hypercalcemia. Although hypercalcemia has been shown to cause QT shortening, in certain instances it may be associated with a normal QT interval $(64,65)$. The QT interval may therefore be an unreliable indicator of the level of hypercalcemia (66). In addition to the effects of $\left[\mathrm{Ca}^{2+}\right]_{0}$ on the RMP and TP, it is possible that the calcium-dependent potassium currents or calcium currents are also altered, and this remains to be studied in the future.

There are several limitations of the present study. First, whilst the mouse is a common animal model for studying cardiac arrhythmias, certain caution must be taken when attempting to extrapolate the results to human findings. In mouse hearts, cardiac action potentials are triangular with the transient outward current $\left(I_{\text {to }}\right)$ being the major repolarizing current (67). By contrast, the human action potential shows a characteristic plateau phase (68). Repolarization is initially mediated by $I_{\text {to }}$, followed by a delayed plateau phase mediated by a balance between the inward calcium current $\left(I_{\mathrm{Ca}}\right)$ and outward delayed rectifier potassium currents $\left(I_{\mathrm{Kr}}\right.$ and $\left.I_{\mathrm{Ks}}\right)$. Guinea pig and rabbit hearts have the same action potential morphology with similar ionic contributions, and may therefore provide better translational results when used as model systems for studying human arrhythmic syndromes (69-80).

Secondly, the MAP technique was chosen for studying electrophysiology in the present study, which allows electrical recordings to be made from intact, isolated, perfused hearts. This has the advantage of preserving intercellular coupling, meaning that the experimental system would be more physiological. MAP recordings close recapitulate the intracellular action potential obtained from single cells $(37,81,82)$. Previous studies have shown that the MAP technique is sufficiently sensitive for detecting alterations in activation latencies, APD and VERP from atrial and ventricular tissue under a variety of experimental conditions (29-32). Furthermore, parameters derived from MAP recordings only show small variations on repeated measurements and between different hearts, suggesting that this technique is sufficiently reliable for studying cardiac electrophysiology. However, certain limitations of the MAP technique must be noted, as recently reviewed (83). One major disadvantage is that MAPs, unlike microelectrode recordings, do not provide information on the upstroke velocity of the cellular action potential. Although the $\mathrm{CV}$ of the propagating excitation wave could not be determined, activation latency could be measured instead. This also permitted the calculation of VERP/latency ratios that are used clinically for approximating the excitation wavelength. Future studies can investigate further by using optical mapping, which would allow simultaneous measurements of cellular activation from numerous recording sites, and CVs as well as excitation wavelength to be calculated. Measurement of magnetic signals, such as cardiac magnetic resonance imaging, has been used for the characterization of structural properties (84-86), and future studies could utilize magnetocardiography to detect electrical abnormalities and predict arrhythmic risk (87-92).

Finally, why epicardial APDs are altered by hyperkalemia whereas endocardial APDs are not remains to be elucidated. Such differences were also observed in experimental 
hypokalemia, where it was noted that epicardial APDs were prolonged but endocardial APDs remained unaltered (40). This may be due to differences in ion channel types or their levels of expression between these regions, but these issues remain to be clarified in future studies.

Taken together, the present study produced an arrhythmia model of hyperkalemia for the first time in the mouse, in which ventricular tachyarrhythmias were associated with shortenings in APD and VERP. Hypercalcemia treatment was able to prevent this arrhythmogenesis through correction of VERP alone without influencing APD, thereby scaling the VERP/latency ratio. Therefore, excitation wavelength appears to be a central determinant of arrhythmogenesis in this system, as has been demonstrated for other models (93).

\section{Acknowledgements}

GT received a Biotechnology and Biological Sciences Research Council (BBSRC) Doctoral Training Award supplemented by Xention Discovery. GT was supported by Xention Discovery. The company did not influence the writing of this manuscript in any manner.

\section{References}

1. No authors listed: Guidelines 2000 for Cardiopulmonary Resuscitation and Emergency Cardiovascular Care. Part 8: Advanced challenges in resuscitation: section 1: Life-threatening electrolyte abnormalities. The American Heart Association in collaboration with the International Liaison Committee on Resuscitation. Circulation 102 (Suppl 8): I217-I222, 2000.

2. Gettes LS: Electrolyte abnormalities underlying lethal and ventricular arrhythmias. Circulation 85 (Suppl 1): I70-I76, 1992.

3. Friedensohn A, Faibel HE, Bairey O, Goldbourt U and Schlesinger Z: Malignant arrhythmias in relation to values of serum potassium in patients with acute myocardial infarction. Int J Cardiol 32: 331-338, 1991.

4. Ahmed MI, Ekundayo OJ, Mujib M, Campbell RC, Sanders PW, Pitt B, Perry GJ, Bakris G, Aban I, Love TE, et al: Mild hyperkalemia and outcomes in chronic heart failure: A propensity matched study. Int J Cardiol 144: 383-388, 2010.

5. Bashour TT and Cheng TO: Evidence for specialized atrioventricular conduction in hyperkalemia. J Electrocardiol 8: 65-68, 1975.

6. Dittrich KL and Walls RM: Hyperkalemia: ECG manifestations and clinical considerations. J Emerg Med 4: 449-455, 1986.

7. Freeman K, Feldman JA, Mitchell P, Donovan J, Dyer KS, Eliseo L, White LF and Temin ES: Effects of presentation and electrocardiogram on time to treatment of hyperkalemia. Acad Emerg Med 15: 239-249, 2008.

8. Cooper WD, Kuan P, Reuben SR and VandenBurg MJ: Cardiac arrhythmias following acute myocardial infarction: Associations with the serum potassium level and prior diuretic therapy. Eur Heart J 5: 464-469, 1984.

9. Weiner ID and Wingo CS: Hyperkalemia: A potential silent killer. J Am Soc Nephrol 9: 1535-1543, 1998.

10. Sood MM and Pauly RP: A case of severe hyperkalemia: Fast, safe and effective treatment is required. J Crit Care 23: 431-433, 2008.

11. Kiewiet RM, Ponssen HH, Janssens EN and Fels PW: Ventricular fibrillation in hypercalcaemic crisis due to primary hyperparathyroidism. Neth J Med 62: 94-96, 2004.

12. Curione M, Letizia C, Amato S, Di Bona S, Di Fazio F, Minisola S, Mazzuoli G and D'Erasmo E: Increased risk of cardiac death in primary hyperparathyroidism: What is a role of electrical instability? Int J Cardiol 121: 200-202, 2007.

13. George SA, Sciuto KJ, Lin J, Salama ME, Keener JP, Gourdie RG and Poelzing S: Extracellular sodium and potassium levels modulate cardiac conduction in mice heterozygous null for the Connexin43 gene. Pflugers Arch 467: 2287-2297, 2015.

14. Kodama I, Wilde A, Janse MJ, Durrer D and Yamada K: Combined effects of hypoxia, hyperkalemia and acidosis on membrane action potential and excitability of guinea-pig ventricular muscle. J Mol Cell Cardiol 16: 247-259, 1984.
15. Leitch SP and Paterson DJ: Role of $\mathrm{Ca} 2+$ in protecting the heart from hyperkalemia and acidosis in the rabbit: Implications for exercise. J Appl Physiol (1985) 77: 2391-2399, 1994.

16. Piktel JS, Wan X, Infeld M, Rosenbaum D and Wilson LD: Beneficial effect of calcium treatment for hyperkalemia is mediated by calcium-dependent conduction, not 'membrane stabilization'. Ann Emerg Med 56: S9, 2010.

17. Tse G and Yeo JM: Conduction abnormalities and ventricular arrhythmogenesis: The roles of sodium channels and gap junctions. Int J Cardiol Heart Vasc 9: 75-82, 2015.

18. Choy L, Yeo JM, Tse V, Chan SP and Tse G: Cardiac disease and arrhythmogenesis: Mechanistic insights from mouse models. Int J Cardiol Heart Vasc 12: 1-10, 2016.

19. Tse G: Both transmural dispersion of repolarization and transmural dispersion of refractoriness are poor predictors of arrhythmogenicity: A role for the index of Cardiac Electrophysiological Balance (QT/QRS)? J Geriatr Cardiol (In press).

20. Tse G, Lai ET, Yeo JM and Yan BP: Electrophysiological mechanisms of Bayés syndrome: Insights from clinical and mouse studies. Front Physiol: May 31, 2016 (Epub ahead of print).

21. Tse G,Lai ET, Lee AP, Yan BP and Wong SH: Electrophysiological mechanisms of gastrointestinal arrhythmogenesis: Lessons from the heart. Front Physiol (In press).

22. Tse G, Lai TH, Yeo JM, Tse V and Wong SH: Mechanisms of electrical activation and conduction in the gastrointestinal system: Lessons from cardiac electrophysiology. Front Physiol: May 31, 2016 (Epub ahead of print).

23. Tse G, Lai ET, Tse V and Yeo JM: Molecular and electrophysiological mechanisms underlying cardiac arrhythmogenesis in diabetes mellitus. J Diabetes Res (In press).

24. Tse G and Yan BP: Novel arrhythmic risk markers incorporating QRS dispersion: QRSd x (Tpeak-Tend)/QRS and QRSdx(Tpeak-Tend)/(QTxQRS).AnnNoninvasiveElectrocardiol (In press)

25. Tse G: Novel conduction-repolarization indices for the stratification of arrhythmic risk. J Geriatr Cardiol (In press).

26. Tse G: (Tpeak-Tend)/QRS and (Tpeak-Tend)/(QT x QRS): Novel markers for predicting arrhythmic risk in Brugada syndrome. Europace (In press).

27. Tse G, Wong ST, Tse V, Lee YT, Lin HY and Yeo JM: Cardiac dynamics: alternans and arrhythmogenesis. J Arrhythm: Mar 28, 2016 (Epub ahead of print).

28. Tse G: Mechanisms of cardiac arrhythmias. J Arrhythm 32: 75-81, 2016.

29. Tse G, Tse V and Yeo JM: Ventricular anti-arrhythmic effects of heptanol in hypokalaemic, Langendorff-perfused mouse hearts. Biomed Rep 4: 313-324, 2016.

30. Tse G, Wong ST, Tse V and Yeo JM: Restitution analysis of alternans using dynamic pacing and its comparison with S1S2 restitution in heptanol-treated, hypokalaemic Langendorff-perfused mouse hearts. Biomed Rep 4: 673-680, 2016.

31. Tse G, Hothi SS, Grace AA and Huang CL: Ventricular arrhythmogenesis following slowed conduction in heptanol-treated, Langendorff-perfused mouse hearts. J Physiol Sci 62: 79-92, 2012.

32. Tse G, Tse V, Yeo JM and Sun B: Atrial anti-arrhythmic effects of heptanol in Langendorff-perfused mouse hearts. PLoS One 11: e0148858, 2016.

33. Hogan PM and Spitzer KW: Manganese amd electrogenic phenomena in canine Purkinje fibers. Circ Res 36: 377-391, 1975.

34. Balasubramaniam R, Chawla S, Mackenzie L, Schwiening CJ, Grace AA and Huang CL: Nifedipine and diltiazem suppress ventricular arrhythmogenesis and calcium release in mouse hearts. Pflugers Arch 449: 150-158, 2004.

35. Balasubramaniam R, Grace AA, Saumarez RC, Vandenberg JI and Huang CL: Electrogram prolongation and nifedipine-suppressible ventricular arrhythmias in mice following targeted disruption of KCNE1. J Physiol 552: 535-546, 2003.

36. Head CE, Balasubramaniam R, Thomas G, Goddard CA, Lei M, Colledge WH, Grace AA and Huang CL: Paced electrogram fractionation analysis of arrhythmogenic tendency in DeltaKPQ Scn5a mice. J Cardiovasc Electrophysiol 16: 1329-1340, 2005.

37. Knollmann BC, Katchman AN and Franz MR: Monophasic action potential recordings from intact mouse heart: Validation, regional heterogeneity, and relation to refractoriness. J Cardiovasc Electrophysiol 12: 1286-1294, 2001.

38. Gussak I, Chaitman BR, Kopecky SL and Nerbonne JM: Rapid ventricular repolarization in rodents: Electrocardiographic manifestations, molecular mechanisms, and clinical insights. J Electrocardiol 33: 159-170, 2000. 
39. Fabritz L, Kirchhof P, Franz MR, Eckardt L, Mönnig G, Milberg P, Breithardt G and Haverkamp W: Prolonged action potential durations, increased dispersion of repolarization, and polymorphic ventricular tachycardia in a mouse model of proarrhythmia. Basic Res Cardiol 98: 25-32, 2003.

40. Sabir IN, Fraser JA, Killeen MJ, Grace AA and Huang CL: The contribution of refractoriness to arrhythmic substrate in hypokalemic Langendorff-perfused murine hearts. Pflugers Arch 454 209-222, 2007.

41. Kléber AG and Rudy Y: Basic mechanisms of cardiac impulse propagation and associated arrhythmias. Physiol Rev 84: 431-488, 2004

42. Thomas GP, Howlett SE and Ferrier GR: Saralasin suppresses arrhythmias in an isolated guinea pig ventricular free wall model of simulated ischemia and reperfusion. J Pharmacol Exp Ther 274: 1379-1386, 1995.

43. Nolan JP, Soar J, Zideman DA, Biarent D, Bossaert LL, Deakin C, Koster RW, Wyllie J and Böttiger B; ERC Guidelines Writing Group: European Resuscitation Council Guidelines for Resuscitation 2010 Section 1. Executive summary. Resuscitation 81: 1219-1276, 2010.

44. Tse G, Wong ST, Tse V and Yeo JM: Depolarization vs. repolarization: What is the mechanism of ventricular arrhythmogenesis underlying sodium channel haploinsufficiency in mouse hearts? Acta Physiol (Oxf): Apr 30, 2016.

45. Chen Z, Sun B, Tse G, Jiang J and Xu W: Reversibility of both sinus node dysfunction and reduced HCN4 mRNA expression level in an atrial tachycardia pacing model of tachycardia-bradycardia syndrome in rabbit hearts. Int J Clin Exp Pathol (In press)

46. Tse G, Wong ST, Tse V and Yeo JM: Determination of action potential wavelength restitution in $\operatorname{Sen} 5 \mathrm{a}+/-$ mouse hearts modelling human Brugada syndrome. J Physiol (In press).

47. Antzelevitch C: Cellular basis for the repolarization waves of the ECG. Ann N Y Acad Sci 1080: 268-281, 2006

48. Fisch C: Relation of electrolyte disturbances to cardiac arrhythmias. Circulation 47: 408-419, 1973.

49. Sanguinetti MC and Jurkiewicz NK: Role of external Ca2+ and $\mathrm{K}+$ in gating of cardiac delayed rectifier $\mathrm{K}+$ currents. Pflugers Arch 420: 180-186, 1992

50. Sanguinetti MC, Jiang C, Curran ME and Keating MT: A mechanistic link between an inherited and an acquired cardiac arrhythmia: HERG encodes the IKr potassium channel. Cell 81: 299-307, 1995

51. Kishida H, Surawicz B and Fu LT: Effects of K+ and K+-induced polarization on (dV/dt)max, threshold potential, and membrane input resistance in guinea pig and cat ventricular myocardium. Circ Res 44: 800-814, 1979.

52. Dominguez G and Fozzard HA: Influence of extracellular K+ concentration on cable properties and excitability of sheep cardiac Purkinje fibers. Circ Res 26: 565-574, 1970.

53. Ettinger PO, Regan TJ and Oldewurtel HA: Hyperkalemia, cardiac conduction, and the electrocardiogram: A review. Am Heart J 88: 360-371, 1974.

54. Killeen MJ, Thomas G, Gurung IS, Goddard CA, Fraser JA, Mahaut-Smith MP, Colledge WH, Grace AA and Huang CL: Arrhythmogenic mechanisms in the isolated perfused hypokalaemic murine heart. Acta Physiol (Oxf) 189: 33-46, 2007.

55. Nerbonne JM and Guo W: Heterogeneous expression of voltage-gated potassium channels in the heart: Roles in normal excitation and arrhythmias. J Cardiovasc Electrophysiol 13: 406-409, 2002

56. Antzelevitch C: Transmural dispersion of repolarization and the T wave. Cardiovasc Res 50: 426-431, 2001.

57. Yan GX and Martin J: Electrocardiographic T wave: A symbol of transmural dispersion of repolarization in the ventricles. J Cardiovasc Electrophysiol 14: 639-640, 2003.

58. Mines GR: On dynamic equilibrium in the heart. J Physiol 46 349-383, 1913

59. Weidmann S: Effects of calcium ions and local anesthetics on electrical properties of Purkinje fibres. J Physiol 129: 568-582, 1955.

60. Hélie F, Cossette J, Vermeulen M and Cardinal R: Differential effects of lignocaine and hypercalcaemia on anisotropic conduction and reentry in the ischaemically damaged canine ventricle. Cardiovasc Res 29: 359-372, 1995.

61. Corlew DS, Bryda SL, Bradley EL III and DiGirolamo M Observations on the course of untreated primary hyperparathyroidism. Surgery 98: 1064-1071, 1985.

62. Rosenqvist M, Nordenström J, Andersson M and Edhag OK: Cardiac conduction in patients with hypercalcaemia due to primary hyperparathyroidism. Clin Endocrinol (Oxf) 37: 29-33, 1992.
63. Parham WA, Mehdirad AA, Biermann KM and Fredman CS Hyperkalemia revisited. Tex Heart Inst J 33: 40-47, 2006.

64. Rumancik WM, Denlinger JK, Nahrwold ML and Falk RB Jr: The QT interval and serum ionized calcium. JAMA 240: 366-368, 1978.

65. Scheidegger D and Drop LJ: The relationship between duration of Q-T interval and plasma ionized calcium concentration: Experiments with acute, steady-state $[\mathrm{Ca}++]$ changes in the dog. Anesthesiology 51: 143-148, 1979.

66. Wortsman J and Frank S: The QT interval in clinical hypercalcemia. Clin Cardiol 4: 87-90, 1981.

67. Killeen MJ, Thomas G, Sabir IN, Grace AA and Huang CL: Mouse models of ventricular arrhythmias. Acta Physiol (Oxf) 192: 455-469, 2008

68. Nerbonne JM and Kass RS: Molecular physiology of cardiac repolarization. Physiol Rev 85: 1205-1253, 2005.

69. Hsieh YC, Lin JC, Hung CY, Li CH, Lin SF, Yeh HI, Huang JL, Lo CP, Haugan K, Larsen BD, et al: Gap junction modifier rotigaptide decreases the susceptibility to ventricular arrhythmia by enhancing conduction velocity and suppressing discordant alternans during therapeutic hypothermia in isolated rabbit hearts. Heart Rhythm 13: 251-261, 2016

70. Wu TJ, Lin SF, Weiss JN, Ting CT and Chen PS: Two types of ventricular fibrillation in isolated rabbit hearts: Importance of excitability and action potential duration restitution. Circulation 106: 1859-1866, 2002.

71. Osadchii OE: Effects of ventricular pacing protocol on electrical restitution assessments in guinea-pig heart. Exp Physiol 97: 807-821, 2012

72. Osadchii OE, Larsen AP and Olesen SP: Predictive value of electrical restitution in hypokalemia-induced ventricular arrhythmogenicity. Am J Physiol Heart Circ Physiol 298: H210-H220, 2010.

73. Osadchii OE: Mechanisms of hypokalemia-induced ventricular arrhythmogenicity. Fundam Clin Pharmacol 24: 547-559, 2010.

74. Osadchii OE, Bentzen BH and Olesen SP: Chamber-specific effects of hypokalaemia on ventricular arrhythmogenicity in isolated, perfused guinea-pig heart. Exp Physiol 94: 434-446, 2009.

75. Osadchii OE and Olesen SP: Electrophysiological determinants of hypokalaemia-induced arrhythmogenicity in the guinea-pig heart. Acta Physiol (Oxf) 197: 273-287, 2009.

76. Osadchii OE: Flecainide attenuates rate adaptation of ventricular repolarization in guinea-pig heart. Scand Cardiovasc J 50: 28-35, 2016.

77. Osadchii OE: Impact of hypokalemia on electromechanical window, excitation wavelength and repolarization gradients in guinea-pig and rabbit hearts. PLoS One 9: e105599, 2014.

78. Osadchii OE: Impaired epicardial activation-repolarization coupling contributes to the proarrhythmic effects of hypokalaemia and dofetilide in guinea pig ventricles. Acta Physiol (Oxf) 211: 48-60, 2014.

79. Osadchii OE: Flecainide-induced proarrhythmia is attributed to abnormal changes in repolarization and refractoriness in perfused guinea-pig heart. J Cardiovasc Pharmacol 60: 456-466, 2012.

80. Hsieh YC, Lin SF, Lin TC, Ting CT and Wu TJ: Therapeutic hypothermia (30 degrees $\mathrm{C}$ ) enhances arrhythmogenic substrates, including spatially discordant alternans, and facilitates pacing-induced ventricular fibrillation in isolated rabbit hearts. Circ J 73: 2214-2222, 2009.

81. Franz MR, Burkhoff D, Spurgeon H, Weisfeldt ML and Lakatta EG: In vitro validation of a new cardiac catheter technique for recording monophasic action potentials. Eur Heart J 7: 34-41, 1986

82. Hoffman BF, Cranefield PF, Lepeschkin E, Surawicz B and Herrlich HC: Comparison of cardiac monophasic action potentials recorded by intracellular and suction electrodes. Am J Physiol 196: 1297-1301, 1959.

83. Tse G, Wong ST, Tse V, Yeo JM, Lin HY and Yeo JM: Monophasic action potential recordings: Which is the recording electrode? J Basic Clin Physiol Pharmacol: Apr 30, 2016 (Epub ahead of print).

84. Tse G, Ali A, Alpendurada F, Prasad S, Raphael CE and Vassiliou V: Tuberculous Constrictive Pericarditis. Res Cardiovasc Med 4: e29614, 2015.

85. Tse G, Ali A, Prasad SK, Vassiliou V and Raphael CE: Atypical case of post-partum cardiomyopathy: an overlap syndrome with arrhythmogenic right ventricular cardiomyopathy? BJRlcase reports 1: 20150182, 2015 . 
86. Vassiliou V, Chin C, Perperoglou A, Tse G, Ali A, Raphael C, Jabbour A, Newby D, Pennell D, Dweck M and Prasad S: 93 Ejection Fraction by Cardiovascular Magnetic Resonance Predicts Adverse Outcomes Post Aortic Valve Replacement. Heart 100 (Suppl 3): A53-A54, 2014.

87. Kwong JS, Leithäuser B, Park JW and Yu CM: Diagnostic value of magnetocardiography in coronary artery disease and cardiac arrhythmias: A review of clinical data. Int J Cardiol 167: 1835-1842, 2013.

88. Steinhoff U, Knappe-Grueneberg S, Schnabel A, Trahms L, Smith F, Langley P, Murray A and Koch H: Magnetocardiography for pharmacology safety studies requiring high patient throughput and reliability. J Electrocardiol 37: 187-192, 2004

89. Yoshida K, Ogata K, Inaba T, Nakazawa Y, Ito Y, Yamaguchi I, Kandori A and Aonuma K: Ability of magnetocardiography to detect regional dominant frequencies of atrial fibrillation. J Arrhythm 31: 345-351, 2015.

90. Ito Y, Shiga K, Yoshida K, Ogata K, Kandori A, Inaba T, Nakazawa Y, Sekiguchi Y, Tada H, Sekihara K, et al: Development of a magnetocardiography-based algorithm for discrimination between ventricular arrhythmias originating from the right ventricular outflow tract and those originating from the aortic sinus cusp: A pilot study. Heart Rhythm 11: 1605-1612, 2014
91. Sato Y, Yoshida K, Ogata K, Inaba T, Tada H, Sekiguchi Y, Ito Y, Ishizu T, Seo Y, Yamaguchi I, et al: An increase in right atrial magnetic strength is a novel predictor of recurrence of atrial fibrillation after radiofrequency catheter ablation. Circ J 76: 1601-1608, 2012.

92. Tse G, Wong ST, Tse V and Yeo JM: Variability in local action potential durations, dispersion of repolarization and wavelength restitution in aged wild-type and Scn5a+/- mouse hearts modelling human Brugada syndrome. Journal of Geriatric Cardiology (In press).

93. Tse G, Yeo JM, Tse V and Sun B: Gap junction inhibition by heptanol increases ventricular arrhythmogenicity by decreasing conduction velocity without affecting repolarization properties or myocardial refractoriness in Langendorff-perfused mouse hearts. MMR (In press).

This work is licensed under a Creative Commons Attribution 4.0 International (CC BY 4.0) License. 